\title{
Correction to: Age differences in Neural Activation to Face Trustworthiness: Voxel Pattern and Activation Level Assessments
}

Yuchen Xie $^{1}$ - John Ksander ${ }^{1}$ - Angela Gutchess ${ }^{1} \cdot$ Nouchine Hadjikhani $^{2,3} \cdot$ Noreen Ward $^{2}$ • Jasmine Boshyan ${ }^{1}$. Leslie A. Zebrowitz ${ }^{1}$

Published online: 28 April 2021

(C) The Psychonomic Society, Inc. 2021

Correction to: Cognitive, Affective, \& Behavioral Neuroscience

https://doi.org/10.3758/s13415-021-00868-y
This article was updated to correct Nouchine Hadjikhani's affiliations.

The online version of the original article can be found at https://doi.org/ $10.3758 / \mathrm{s} 13415-021-00868-\mathrm{y}$

\section{Leslie A. Zebrowitz}

zebrowit@brandeis.edu

1 Brandeis University, Waltham, MA, USA

2 Athinoula A Martinos Center for Biomedical Imaging, Radiology, Charlestown, MA, USA

3 Gillberg Neuropsychiatry Center, University of Gothenburg, Sahlgrenska Academy, Kungsgatan 12, SE-411

19 Göteborg, Sweden 